\title{
Germline genomes have a dominant-heritable contribution to cancer immune evasion and immunotherapy response
}

\author{
Xue Jiang ${ }^{1, \dagger}$, Mohammad Asad ${ }^{2, \dagger}$, Lin $\mathrm{Li}^{2}$, Zhanpeng Sun ${ }^{2,3}$, Jean-Sébastien Milanese ${ }^{4}$, Bo Liao ${ }^{5, *}$, \\ Edwin Wang ${ }^{2, *}$ \\ 1 Shanghai Mental Health Center, School of Biomedical Engineering, Shanghai Jiao Tong University, Shanghai 200030, China \\ 2 Department of Biochemistry and Molecular Biology, Cumming School of Medicine, University of Calgary, Calgary, T2N 4N1, \\ Canada \\ 3 Tianjin Normal University, Tianjin 300074, China \\ ${ }^{4}$ Human Health Therapeutics Research Centre, National Research Council Canada, Montreal, H4P 2R2, Canada \\ ${ }^{5}$ School of Mathematics and Statistics, Hainan Normal University, Haikou 570206, China \\ * Correspondence: edwin.wang@ucalgary.ca, dragonbw@163.com
}

Received January 22, 2020; Revised March 28, 2020; Accepted April 30, 2020

Background: Immune evasion is a fundamental hallmark for cancer. At the early stages of tumor development, immune evasion strategies must be implemented by tumors to prevent attacks from the host immune systems. Blocking tumors' immune evasion will re-activate the host immune systems to eliminate tumors. Immune-checkpoint therapy (ICT) which applies anti-PD-1/PD-L1 or anti-CTLA4 treatment has been a remarkable success in the past few years. However, $\sim 70 \%$ of patients cannot gain any clinical benefits from ICT treatment due to the tumorimmunity system's complexity. In the past, germline pathogenic variants have been thought to have only minorheritable contributions to cancer.

Results: Emerging evidence has shown that germline genomes play a dominant-heritable contribution to cancer via encoding the host immune system. The functional components of the immune system are encoded by the host genome, thus the germline genome might have a profound impact on cancer immune evasion and immunotherapy response. Indeed, recent studies showed that germline pathogenic variants can influence immune capacity in cancer patients at a population level by (i) shaping tumor somatic mutations, altering methylation patterns and antigen-presentation capacity or (ii) influencing NK cell's function to modulate lymphocyte infiltration in the tumor microenvironment. In addition, the HLA (types A, B or C) genotypes also shape the landscape of tumor somatic mutations.

Conclusion: These results highlight the indispensable roles of germline genome in immunity and cancer development and suggest that germline genomics should be integrated into the research field of cancer biology and cancer immunotherapy.

Keywords: germline; genomics; cancer; immune evasion; immunotherapy response

Author summary: Traditionally, it has been believed that germline pathogenic variants and family histories explain $5 \%-10 \%$ of cancer patient population, thus, heredity has been suggested to have a small contribution to tumorigenesis and metastasis. However, the host immune system often interacts with cancer cells, therefore, escaping from the host immune system surveillance is one of the critical means for tumorigenesis. In the past a few years, it has been shown that germline pathogenic variants influence immune capacity in cancer patients at a population level. From the cancer-immune system point view, heredity plays a dominant role in tumorigenesis, metastasis and immunotherapy response.

$\bar{\dagger}$ These authors contributed equally to this work. 


\section{INTRODUCTION}

\section{Immune evasion is a fundamental hallmark for cancer cell survival}

One of the cancer hallmarks is immune evasion [1]. During the initiation and development of tumors, the host immune system monitors "foreign" cells such as cancerous cells in the human body. Generally, the immune system can eliminate these mutated cells, however, these cells can find ways to escape from the host immune system by developing various immune evasion strategies. For example, tumor cells can deregulate pathways such as antigen presentation [2] and interferon-gamma (IFN- $\gamma$ ) pathways [3] or activate intrinsic signaling pathways such as mitogen-activated protein kinase (MAPK) $[4,5]$ and Wnt signaling pathways $[6,7]$ to block tumor-infiltrating lymphocytes (TILs) from entering the tumor immune microenvironment (TIME). Without implementing these immune evasion strategies, a tumor cell can be quickly removed by the host immune system.

Immunotherapy represents a truly exciting therapeutic success in oncology. Particularly, the discovery of immune "brakes" or checkpoint proteins, such as cytotoxic T-lymphocyte-associated protein 4 (CTLA4), programmed cell death protein-1 (PD-1) and programmed cell death protein ligand-1 (PD-L1), has led to developing of antibodies to inhibit these checkpoints which could further remove the immune "brakes", allowing $\mathrm{T}$ cell activation and elimination of tumors. In the past few years, ICT has shown an overall response rate of $10 \%-$ $40 \%$ in patients with melanomas and other types of cancer $[8,9]$. However, for the rest of these patients, ICT failed to show their intended effect.

ICT response is dependent on many factors. For example, tumor antigens need to be released and presented onto the tumors' cell surface so that immune cells such as $T$ cells will be activated and infiltrated into the tumor microenvironment. Further, anti-PD-1/PD-L1 or anti-CTLA4 treatment helps in overcoming an immunosuppressive microenvironment for cytotoxic $\mathrm{T}$ lymphocytes (CTLs) to recognize and ultimately kill these tumor cells [10]. TIME interacts with cancer cells to influence metastasis and clinical outcomes [11,12]. Therefore, a deeper understanding of TIMEs and identification of key germline regulators modulating TIMEs and immune-evasion strategies would shed further insights into ICT response, resistance and might improve existing immunotherapies. For example, it has been known that rich TILs are critical for ICT response [13], and thus understanding how TILs are affected by germline genomics will provide a new avenue for ICT treatment. Due to the relatively short period of a wide application of immunotherapy, the roles of germline genomics in cancer immunity and immunotherapy have not been extensively studied. Nevertheless, several pilot studies have been conducted in this direction. In this review, we will discuss the recent progress and its implications for future research in this area.

\section{Germline pathogenic variants have been shown to have only minor-heritable contributions in cancer in the past}

Traditionally, inherited germline variants have been shown to account for a relatively small fraction of cancer patients. For example, the most famous $B R C A 1 / 2$ germline pathogenic variants account for $5 \%-10 \%$ of breast or ovarian cancer $[14,15]$. The effect sizes of other germline variants are relatively small, and thereby have a minor influence on cancer development [16]. Besides, many germline variants are haplotypes and thus do not have a significant impact on cancer development as well. Albert Knudson was the first scientist who discovered germline variants in 1971. He studied patients with retinoblastoma carrying mutations in RB1. At that time, it was already known that two alleles existed for each gene, and a mutation in both copies of RB1 would be required to develop retinoblastoma. Knudson noticed that bilateral retinoblastomas (i.e., retinoblastoma in both eyes) were more frequent in younger patients $(25 \%-30 \%$ of all cases), suggesting that germline pathogenic variants were involved. As mutations in both alleles of RB1 are required to develop retinoblastoma, inherited germline mutation in this gene was predisposing the children to cancer. Knudson's findings were later described as the "twohit" hypothesis, supposing that children possessing an inherited RB1 mutation need to obtain a second mutation to develop retinoblastoma $[17,18]$. In contrast, children without RB1 pre-disposition were less likely to develop retinoblastoma as they needed to acquire two mutations. Mice with germline inactivation in Rb1 and p107 (another $\mathrm{Rb}$ family member) developed unilateral retinoblastoma by nine months [19], while with germline deficiency in $\mathrm{Rb} 1$ and p130 (another Rb family member) developed bilateral retinoblastomas with a $100 \%$ of penetrance in four months [20]. Today, multiple hereditary germline mutations have been associated with cancer. For example, people carrying a dysfunctional germline $\mathrm{p} 53$ gene have a $\sim 90 \%$ chance of developing a variety of cancers before the age of 70 [21]. Similarly, mice deficient of germline p53 are extremely susceptible to radiation-induced tumorigenesis [22,23]. p53 is widely regarded as the most important tumor suppressor in the human genome. It acts as one of the major gatekeepers in cancer and has multiple functions such as ensuring genomic integrity and 
promoting DNA repair, cell cycle regulation and apoptosis, signal transduction and cell adhesion [24]. Other cancer-related inherited germline pathogenic variants include those found in $B R C A 1 / 2$, which are tumor suppressor genes involved mainly in DNA repair and are associated with breast and ovarian cancer $[25,26]$. In addition, germline pre-dispositions in BRCA1/2 in women have been associated with an increased risk of developing breast and ovarian cancer by the age of $80(69 \%-72 \%$ and $17 \%-44 \%$, respectively) [27]. Although the prevalence of $B R C A 1 / 2$ germline pathogenic variants worldwide is very low $(5 \%-10 \%)$, they are now regularly checked in genetic screening. Other heritable genes include PTEN, whose germline mutations induce Cowden syndrome [28], and APC, which has been associated with familial adenomatous polyposis [29] and is accurately modeled by germline mutations in mouse models [30,31]. RET and MEN1 germline mutations have been shown to cause endocrine cancers while $V H L$ alterations result in kidney and other types of cancer [32]. Moreover, Lynch syndrome, a form of colorectal cancer, is linked to germline mutations in $M S H 2, M L H 1, M S H 6, P M S 2$ and EPCAM [33]. A lot of these germline mutations could be or have been modeled by experimental approaches such as using CRISPR/Cas9-modified mice [34,35]. Investigation of whole exomes in cancer patients revealed that the germline genomic landscape of these patients is informative and can provide insights on tumor development $[36,37]$. However, these germline variants have relatively low penetrance and can only explain the heredity for $5 \%-$ $10 \%$ of cancer patients [37,38]. Therefore, germline genomic information has been largely ignored in cancer research due to its limited role in cancer development in the past.

\section{GERMLINE PATHOGENIC VARIANTS MODULATING CANCER IMMUNE EVASION AND IMMUNOTHERAPY RESPONSE ARE HERITABLE ACROSS THE WHOLE CANCER PATIENT POPULATION}

As shown above, germline pathogenic variants are thought to have only minor contributions to cancer, and thus have been largely ignored in cancer research. As systems biology emerges and develops in the past 10 years, it is now possible to identify germline variants which encode proteins in meaningful processes such as the immune system that could have a heritable influence on cancer development. In the cancer hallmark network framework [39], we proposed the logics of complex cancer biology as a myriad of phenotypic complexities governed by a limited set of underlying organizing principles. Thus, we expected that germline pathogenic variants in some of these organizing principles (or cancer hallmarks) could have a heritable influence across the whole cancer patient population, and thus play a dominant-heritable role in cancer development and metastasis. In particular, in the cancer-immunity system, the immune components are not so heterogeneous as tumor cells are, therefore, germline pathogenic variants related to the immune system could have a heritable influence on immune evasion and immunotherapy dominantly. The role of germline pathogenic variants on the cancer-immunity system has been rarely investigated. Recently, we and others pursued this direction using omics data collected from The Cancer Genome Atlas (TCGA) and showed that germline pathogenic variants influence cancer-immunity and immunotherapeutic response via different mechanisms (Fig. 1).

Germline genomes provide evolutionary and immune constraints to tumor somatic mutations across the whole cancer patient population

Although cancer somatic mutations seem chaotic, complex and unorganized, they have been selected by the host-specific genetic makeup to become the components of cancer cell machinery representing cancer hallmarks [39]. In the cancer hallmark network framework [39], we have proposed that the pre-existing genetic makeup could restrain somatic mutations and cancer clonal evolution to form specific signaling pathways and regulatory networks to represent cancer hallmarks. Thus, the cancer hallmark network framework suggests that based on host germline genomic information, a cancer patient's somatic mutations in his/her tumors are predictable, and therefore, the tumor recurrence and metastasis are also predictable.

To demonstrate these concepts, we have developed a novel network-based computational algorithm, eTumorMonitor, which predicts key somatic mutating genes based on host genomic information (Tibiche et al., unpublished data). By applying this algorithm to the germline whole-exome genomic data of breast, lung, and prostate cancer patients, we were able to predict 20-30 key somatic mutating genes in the cancer founding clone, which is the first cancer cell inferred from tumor sequencing data, for each patient. The predicted somatic mutating genes were enriched 10,000 times comparing to random groups. The prediction applies to all the cancer patients tested ( $n>600$, Fig. 1). These results support the notion in the cancer hallmark network framework that host pathogenic variants play an important role in selecting somatic mutating genes during cancer clonal evolution. These key somatic mutating genes in tumor cells will be organized into several tumor cell-intrinsic 


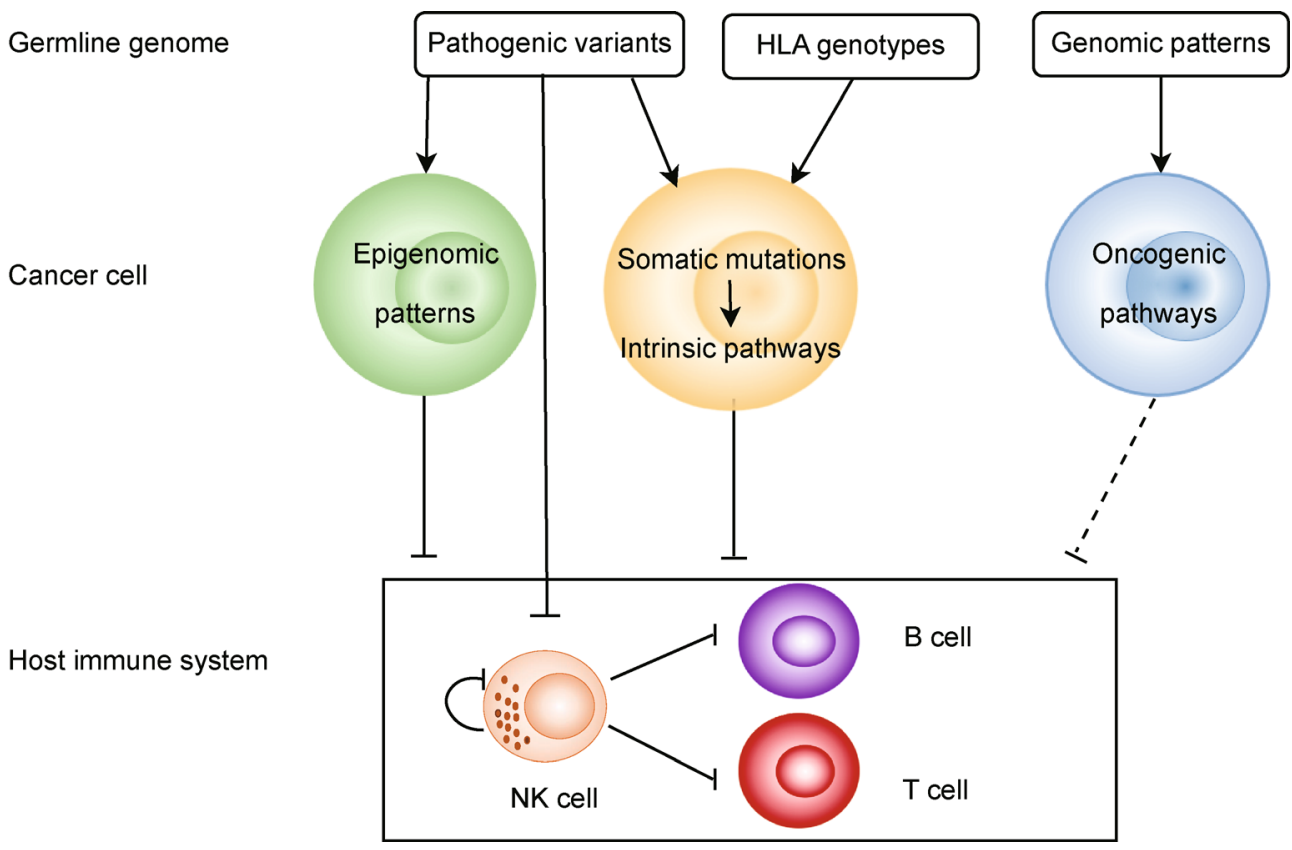

Figure 1. Germline genomes have a dominant-heritable contribution to cancer-immunity. The landscape of tumor somatic mutations can be shaped by both germline pathogenic variants (i.e., via functional restraints) and HLA genotypes (i.e., via immune selections), which further influence tumor initiation, progression, and recurrence. Germline pathogenic variants negatively regulate cancer-immunity, restraining TILs from entering the TIME and compromising ICT effects by altering tumor intrinsic signaling pathways, regulatory networks or epigenomic patterns. Genomic patterns, or significantly repeatedly occurring sequential mutation profiles, of cancer patients, have a significant impact on oncogenic pathways and tumor metastasis and have a negative regulation for the immune system. Germline pathogenic variants in NK cells in cancer patients are negatively correlated with TILs' abundance in TIMEs, immunotherapy response and clinical outcomes. An arrow represents a positive regulation while a delimiter represents a negative regulation.

signaling pathways to maintain the malignant growth of cancer. Key tumor cell-intrinsic signaling pathways such as MAPK, Wnt and TGF- $\beta$ pathways are associated with cell proliferation and metastasis [40]. Also, recent works showed that blocking TILs in TIMEs is an intrinsic feature for germline pathogenic variants [41-43]. Thus, host germline genomes could regulate TIMEs and immunotherapy response by selecting key cancer somatic mutating genes, pathways and regulatory networks. Rethinking of the constraints on cancer somatic mutations by host-specific genetic makeup would provide a novel window to explore and understand the complexity of tumor-host interactions.

Except for the functional constraints exerted on cancer somatic mutations by germline genomes, as shown above, HLA genotypes can exert an immune constraint on the landscape of tumor somatic mutations as well (Fig. 1). Major histocompatibility complex (MHC) class I (HLAA, -B and -C) is an important machinery for antigen presentation of peptides for intracellular proteins including cancer-driving proteins onto the cell surface. $\mathrm{CD} 8^{+} \mathrm{T}$ cells can recognize "foreign antigens" which are derived from mutated proteins and thus kill the cancer cells. The binding affinity of the MHC-I complex for peptides is a major determinant of antigenicity: if a peptide can bind to the MHC-I with a higher binding affinity, it can be easier to be presented onto the cell surface so that $\mathrm{T}$ cell can recognize it. MHC-I binding affinity is dependent on the genotypes of three HLA genes (HLA-A, HLA-B and HLA-C) which are highly polymorphic. Thus far, more than 10,000 HLA alleles for the three genes have been documented [44]. The combination of six MHC-I alleles in each person determines which peptides can be more effectively presented. Thus, each person may have an MHC-I complex that possesses different binding affinity to a same peptide. If a mutated peptide can be effectively presented, the corresponding cell can be eliminated by $\mathrm{T}$ cells during the early stages of tumor development. A computational analysis of the somatic mutations/peptides in thousands of tumors has shown that MHC-I genotype influences the occurrence of specific oncogenic mutations, and recurrent oncogenic mutations are enriched in the peptides that are poorly presented by MHC-I complexes [45]. Somatic mutations encoding poorly 
presented peptides were more frequently observed in tumors [45]. The restriction on the landscape of cancer somatic mutations in tumors by MHC-I genotypes is not limited in a subset of patients but actually in almost all patients we tested (i.e., several thousands of cancer patients collected in TCGA) [45]. These results suggest that germline HLA-A, HLA-B, and HLA-C variants restrict cancer somatic mutations via immunoediting during tumor formation. One interesting question remains: are people who have certain HLA genotypes having a higher risk of developing solid cancer?

It should be noted that one drawback of this study [45] is that they used all the somatic mutations in the tumors to examine the immune-selection pressures from the HLA phenotypes. Based on the timing of individual tumor evolution, their somatic mutations can be classified into two groups: founding clonal mutations and subclonal mutations. Genomic and epigenetic alterations in the process of transformation of a normal cell into a cancer founder cell in a tumor are called founding clonal mutations or alterations, while the rest are called subclonal mutations or alterations [39,46-48]. While there are a few different opinions $[49,50]$, the theory that tumor originates from one founding clone is commonly accepted and validated by many studies [51-58]. We hypothesized that immune-escaping strategies exist before the formation of a founding clone for each tumor. As mentioned above, these strategies could include constraining somatic mutations through antigen presentation pathway (related to HLA gene polymorphism), deregulating TILs in TIMEs through INF- $\gamma$, MAPK and Wnt pathways, or altering expression of PD-1/PD-L1 and CTLA4 checkpoint proteins. Therefore, once a founding clone is formed, the immune-selection pressure on somatic mutations from the MHC-I system could be dramatically reduced or even disappeared, meaning that the immune-selection pressure on somatic mutations from the MHC-I system could be much less in subclones. Nevertheless, selection pressure from HLA genotypes could largely play its role in the early stages of cancer development, namely, before the formation of a founding clone. These immune evasion strategies may shape signaling pathways in cancer founding clones, which in turn, influence the signaling pathways in their subclones by functionally selecting somatic mutations to enhance cancer hallmarks [39].

\section{Germline genomes influence tumor recurrence via immune-related genes}

To further prove the correctness of these concepts proposed in the cancer hallmark network framework mentioned above, we have developed another computa- tional algorithm, eTumorMetastasis [59], which transforms germline pathogenic variants into network-based profiles and identifies network operational gene signatures. These gene signatures model the tipping point at which a tumor cell shifts from a state that does not favor recurrences to one that does. In addition, to improve prediction accuracies and robustness, eTumorMetastasis incorporates key concepts of the multiple survival screening (MSS) algorithm [60,61] developed by our lab previously. By applying the eTumorMetastasis to whole-exome germline genomic sequencing data of $\mathrm{ER}^{+}$ breast cancer patients [48], we showed that gene signatures derived from the genes encoding germline pathogenic variants significantly distinguished recurred and non-recurred patients in $\mathrm{ER}^{+}$breast cancer cohorts ( $n=200$ and 295, respectively, Fig. 1). Furthermore, the germline-based prediction significantly outperforms the well-known Oncotype DX test (i.e., Oncotype DX breast cancer recurrence test) which is widely used in clinics to help doctors making decisions for clinical treatments by determining the probability of recurrence based on the expression of 21 genes in the Oncotype DX 21-gene assay in breast tumors [62]. It should be noted that our predictions are based on the whole $\mathrm{ER}^{+}$breast cancer population, suggesting that host germline pathogenic variants could provide a profound constraint on the evolution of tumors and therefore exert a contingent effect on tumor metastasis and clinical outcomes. The performance of our algorithm (eTumorMetastasis) [48,59] strongly supports the notion proposed in the cancer hallmark network framework [39]. Therefore, we proposed that germline genomic information of cancer patients could predict cancer recurrence in a populationwide manner [59].

Practically, eTumorMetastasis provides a new possibility for developing blood tests to predict prognosis in different types of cancer. Traditionally, the prediction of tumor recurrence (such as the Oncotype DX assay) is based on sequencing data of tumor samples. To conduct the test using tumor samples, an invasive, often risky and painful procedure is used, either through a needle or surgery. However, our eTumorMetastasis-based test is utilizing "liquid biopsy", which uses white cells from blood $[48,59]$.

In addition, germline pathogenic variants are predominantly enriched in the processes related to $\mathrm{T}$ cell function, antigen presentation and cytokine interactions [43], suggesting that inherited germline variants are likely impairing the adaptive and innate immune systems in recurred samples. These results provide a new angle to understand the host-tumor interaction that germline pathogenic variants of immune-related genes could play an important role in metastasis. 


\section{Germline genome sequence patterns shape oncogenic pathways, cancer risk, and tumor recurrence}

Genome sequence can be a "dark matter" which encodes information related to phenotypes, however, lots of the encoded information in human genomes are not wellunderstood. To explore if germline genome sequences relate to cancer occurrence and clinical outcomes, we analyzed genomic patterns, or significantly repeatedly occurring sequential mutation profiles, in the germline genomes of cancer patients $(n=9,712)$ representing 22 common cancer types along with non-cancer individuals $(n=16,670)$. We showed that seven germline genomic patterns could be associated with carcinogenesis, cancer risk and cancer clinical outcomes [63]. One of the genomic patterns was significantly enriched in the germline genomes of patients who smoked compared to non-smokers in the 13 most common cancer types, suggesting that this germline genomic pattern was predisposed to carcinogenesis in patients who smoke. The several genomic patterns in germline genomes shape the landscape of somatic mutations of key oncogenic genes, relate to oncogenic pathways and prognosis in the 12 common cancer types, suggesting that germline genomic patterns can predict clinical prognosis [63]. These results demonstrate that cancer risk and clinical outcomes could be predicted by the specific germline genomic patterns in germline genomes (Fig. 1), and provide a novel perspective for further investigation if these patterns have an impact on ICT response.

\section{Germline pathogenic variants of immune cells shape TIMEs and immunotherapy response}

TIME plays a critical role in metastasis and immunotherapy [11]. Without TILs, tumors have a weak response to ICT. To identify key regulators that define TILs' abundance in TIMEs, we classified $\sim 6,000$ tumors from TCGA representing 13 common cancer types into TIMErich, -intermediate and -poor subgroups which contain high, intermediate or low levels of TILs, using tumor RNA-seq data and by clustering analysis [64]. Analysis of these three subtypes indicated that TIME-rich tumors have a significantly better prognosis than TIME-poor tumors. By applying the TIME subtypes to ICT clinical trial samples, we showed that anti-PD-1 responders were significantly enriched in TIME-rich tumors, while almost none were found in TIME-poor tumors [64]. Genomewide analyses of germline variants in the 6,000 patients showed that inherited germline pathogenic variants in natural killer (NK) cells are significantly associated with the TIME-poor subtype [64]. The number of inherited germline pathogenic variants in NK cells are negatively associated with TILs' abundance, metastasis, survival and ICT response, but positively correlated with the risk of developing cancers [64]. These results suggest that germline variants in NK cell-related genes not only affect NK cells, but also affect other types of immune cells (such as T cells) from entering the TIMEs, and thus increase the risk of developing tumors. Notably, the conclusions are drawn from $\sim 6,000$ cancer patients representing 13 common cancer types, suggesting that germline pathogenic variants in NK-related genes play an important role in modulating TILs' abundance in TIMEs, ICT response and clinical outcomes in a dominant manner (Fig. 1).

\section{Germline variants shape DNA methylation patterns and TIMEs in tumors}

Other than genomes, the epigenome provides another layer of gene regulation on transcriptional programs, which impacts phenotypes and cellular memory. Epigenomic changes have been well-documented in cancer development [65]. Somatic mutations in genes such as chromatin-remodeling factors [66], histone-modifying enzymes [67], and DNA methylation related enzymes [68] have also been found in tumors. By comparing differentially expressed genes between TIME-rich and TIME-poor tumors in TCGA, we found that chromatinremodeling related genes are significantly associated with TIME-poor tumors (unpublished data), suggesting that deregulated chromatin landscape of tumors plays an important role in modulating the immune landscape and TIMEs. Activated SWI/SNF chromatin remodeling complex in tumor cells inhibits the activation of IFN-pathway $[69,70]$. Inactivation of IFN-pathway to reduce IFN- $\gamma$ production is one of the common immune evasion strategies for tumors [71,72]. Furthermore, the deletion of PBRM1, a subunit of the SWI/SNF chromatin remodeling complex, increased response rates to antiPD-1/PD-L1 treatment in cell renal cell cancer patients $[69,70]$. These results suggest that the deregulation of DNA methylation programs plays a key role in the regulation of TIMEs and ICT response. It should be noted that epigenetic modifications are often reversible, and thus it is promising to improve ICT responses via epigenetic interference. A recent study showed that germline variants in prostate cancer patients have an impact on tumor epigenetic profiles: many germline variants influence methylation levels in prostate tumors but not in normal tissues [73], in a population-wide manner (Fig. 1). These results suggest that germline variants modulate DNA methylation and tumor epigenome to contribute to tumorigenesis and metastasis. Prostate cancer is a highly inheritable cancer [74], therefore, the links between germline variants and tumor epigenome in prostate cancer may not apply to other cancers. 


\section{GERMLINE PATHOGENIC VARIANTS MODULATE CANCER IMMUNE EVASION AND IMMUNOTHERAPY RESPONSE IN SUBSETS OF CANCER PATIENTS}

Except for the population-wide influence of cancer immune-evasion and immunotherapy response by germline pathogenic variants, it has been found that some germline genetic factors have an impact on cancer immune-evasion and immunotherapy response in subsets of patients.

\section{Germline variants in DNA-repair machinery genes regulate immunotherapy response}

Dysfunction in DNA-repair machinery is another important contributing factor for cancer development, progression, and metastasis [75]. For a small fraction of cancer patients, their germline genomes contain pathogenic variants in the DNA repair genes, which lead to increased genome instability. Genome instability is a major driving force for increasing somatic mutations in tumor cells [39]. If a tumor has more somatic mutations, it might have more neoantigens being presented onto the cell surface and thus have a higher chance of responding to ICT. Several studies have shown that tumor mutational burden (TMB) is positively correlated with the response rate of ICT [76]. Currently, TMB has been used as a biomarker to predict ICT response [77], although the accuracy is not very high.

\section{Germline variants in chromosomal instability genes contribute to cancer immune evasion}

Chromosomal instability (CIN) is another hallmark for cancer [1]. Through an analysis of germline genomes of cancer patients from TCGA, we found that CIN is significantly enriched in the germline genomes of a small fraction of cancer patients (of 12 common cancer types) than that of non-cancer individuals (unpublished data). These results agree with the estimation that $60 \%-80 \%$ of human tumors exhibit chromosomal abnormalities [78,79]. Chromosome segregation errors (i.e., for CIN) can activate innate immune signaling through the introduction of genomic double-stranded DNA (dsDNA) into the cytosol and engagement of the cGASSTING cytosolic dsDNA-sensing, anti-viral pathway $[80,81]$. This might explain CIN's role in immune evasion, tumor evolution, and metastasis.

\section{Germline variants in immune-related genes affect ICT response}

In current ICT, CTLA4 is one of the key immune checkpoints. Inherited germline pathogenic variants in
CTLA4 gene display a different response to ICT compared to wild-type CTLA4 [82,83]. In another study, it was found that germline mutations in CTLA4 would decrease numbers as well as functions of Tregulatory (Treg) cells, and lead to hyperproliferation of $\mathrm{T}$ cell and a progressive decrease in B cells [84]. Germline pathogenic variants in genes related to interleukins and chemokines show a different response to both anti-PD-1 and anti-CTLA4 treatments [85]. IFN signaling pathway is critical for tumor cells escaping. Germline pathogenic variants in IFN regulatory factor-5 (IRF5) are associated with different efficacy to immunotherapy $[86,87]$. Fc- $\gamma$ receptors $(\mathrm{Fc} \gamma \mathrm{Rs})$ are found on the surface of many immune cells such as NK cells and B cells, and function in regulating the immune system. Germline pathogenic variants in the $\mathrm{Fc} \gamma \mathrm{R}$ gene compromised the efficacy of anti-CTLA4 treatment [88]. Germline pathogenic variants that could activate JAK3 led to increased expression of PD-L1 on both tumor cells and macrophages [89]. These results implied that germline variants in immune-related genes affect ICT response.

Germline pathogenic variants in the genes of immune cells modulate immune-evasion and ICT response

The human immune system includes more than 20 types of immune cells which are key members of innate and adaptive immune components and signaling molecules such as chemokines and cytokines. Germline pathogenic variants of TLR4, P2RX7, and FPR1 may modulate antitumor immune response [90,91]. Germline variants in genes encoding chemokines and their receptors, cytokines or molecules in IFN pathway, such as IL-10, CCL5, JAK, and IFNGR, could modulate the recruitment of immune cells into TIMEs [92-95]. Germline variants in HLA-E, which is recognized by specific NK cell receptors, affect ICT response [96]. Also, germline variants in genes in immune cells such as T cells, B cells, NK cells or macrophages could modulate immune-evasion and ICT response [64] (unpublished results).

\section{IMPLICATIONS AND FUTURE DIRECTIONS}

It is both interesting and unexpected that recent studies highlight an important role of germline genomes in regulating cancer immunity and immunotherapy response. The germline genome can restrict cancer somatic mutations through either HLA genotypes or functional/evolutionary constraints in a population-wide manner. In addition, germline genomes could encode genetic factors that shape tumor epigenomic events, oncogenic pathways, tumor recurrence, and cancer risk by 
modulating cancer immunity. Moreover, germline variants regulate functions of immune cells such as NK cells to modulate cancer immunity and ICT response. The uncovering of the causal relationships between germline genomes, epigenome, somatic mutations in tumors and NK cell variability for regulating TILs in TIMEs would provide new avenues such as reprogramming TIMEs for cancer treatment.

\section{Resolving of a long-term debate in cancer biology}

It has been debated for many years whether genetic, extrinsic factors [97] (i.e., exposure to pathogen infection and environmental agents contributes to tumorigenesis) or intrinsic factors [98] ("bad luck hypothesis" - random error mutations during DNA replication in some stem cells could explain the majority of cancer population) contribute to tumorigenesis and metastasis. Although both "bad luck" and "extrinsic factor" hypotheses disagree with each other in terms of intrinsic and extrinsic factors for contributing tumorigenesis, they agree that heredity plays a minimal role in tumorigenesis. Indeed, from the cancer cell perspective, family history and a handful of known germline pathogenic variants may only explain less than $5 \%$ of cancer cases. Based on systems biology, in the cancer hallmark network framework, we proposed that pre-existing genome (i.e., in-born genome or germline genome) could play a dominant-heritable role in shaping tumorigenesis and metastasis [39]. The key limitation of both "bad luck" and "extrinsic factor" hypotheses is that they are cancer cell-centered and ignored the variance of the host immune system. Here we and others have shown that germline pathogenic variants play a significant role in the cancer-immunity system (Fig. 2), highlighting that heredity does not play a minimal, but dominant role in tumorigenesis. In summary, from the view of cancer cells, heredity plays a minimal role in tumorigenesis, however, from the view of the cancer-immunity system, heredity plays a critical role in both tumorigenesis and metastasis.

\section{The necessity to integrate germline genomic information into the personalized immunotherapy}

In the past, germline pathogenic variants have been ignored in both cancer research and clinics. With the new findings of germline genetic contributions to cancerimmunity, the factors influencing ICT response should be considered in an integrated manner - both germline and somatic mutations (including other genomic and epigenetic alterations such as DNA methylation) should be associated with clinical outcomes. From the evidence above, germline genetic background might affect the degree of tumor immune heterogeneity (TILs and TIMEs in tumors) and then affect ICT response. We have shown that germline pathogenic variants are able to predict prognosis, thus, it would be critical to integrate germline data with tumor data such as gene expression, genomic
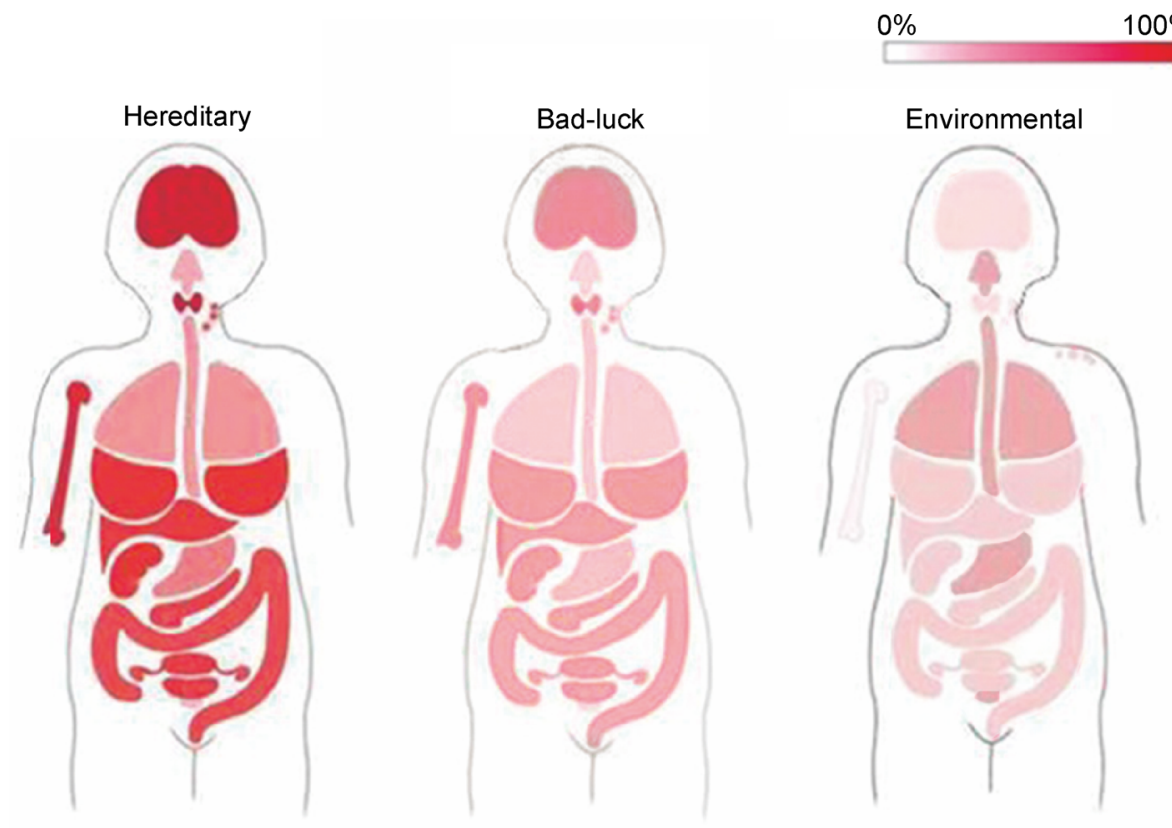

Figure 2. Contributions of germline genomes (i.e., heredity), bad luck (i.e., replicate) and external factors (i.e., environmental) to tumorigenesis and metastasis. The degree of contributions for each factor to tumorigenesis and metastasis in each organ (bone, bone marrows, brain, thymus, respiratory tract, lung, stomach, colon, kidney, liver, ovary, and urinary system) is illustrated by the color depth (from light pink to deep red). 
and epigenetic alterations to construct machine learning models to select patients for immunotherapy.

Both research and clinical applications of immunity in cancer are still in the early phase. Lots of questions remain unanswered. For example, how many cancer immune evasion strategies can tumors use? Does a tumor utilize only one or several immune-evasion strategies? Which are the key immune evasion mechanisms that might be modulated by germline pathogenic variants? There are more questions which need to be asked and answered.

\section{COMPLIANCE WITH ETHICS GUIDELINES}

The authors Xue Jiang, Mohammad Asad, Lin Li, Zhanpeng Sun, JeanSébastien Milanese, Bo Liao and Edwin Wang declare that they have no conflict of interests.

This article is a review article and does not contain any studies with human or animal subjects performed by any of the authors

\section{REFERENCES}

1. Hanahan, D. and Weinberg, R. A. (2011) Hallmarks of cancer: the next generation. Cell, 144, 646-674

2. Kriegsman, B. A., Vangala, P., Chen, B. J., Meraner, P., Brass, A. L., Garber, M. and Rock, K. L. (2019) Frequent loss of IRF2 in cancers leads to immune evasion through decreased MHC class I antigen presentation and increased PD-L1 expression. J. Immunol., 203, 1999-2010

3. Zhang, S., Kohli, K., Black, R. G., Yao, L., Spadinger, S. M., He, Q., Pillarisetty, V. G., Cranmer, L. D., Van Tine, B. A., Yee, C., et al. (2019) Systemic interferon- $\gamma$ increases MHC class I expression and T-cell infiltration in cold tumors: results of a phase 0 clinical trial. Cancer Immunol. Res., 7, 1237-1243

4. Lu, Y., Zhang, M., Wang, S., Hong, B., Wang, Z., Li, H., Zheng, Y., Yang, J., Davis, R. E., Qian, J., et al. (2014) p38 MAPK-inhibited dendritic cells induce superior antitumour immune responses and overcome regulatory T-cell-mediated immunosuppression. Nat. Commun., 5, 4229

5. Loi, S., Dushyanthen, S., Beavis, P. A., Salgado, R., Denkert, C., Savas, P., Combs, S., Rimm, D. L., Giltnane, J. M., Estrada, M. V., et al. (2016) RAS/MAPK activation is associated with reduced tumor-infiltrating lymphocytes in triple-negative breast cancer: Therapeutic cooperation between MEK and PD-1/PD-L1 immune checkpoint inhibitors. Clin. Cancer Res., 22, 1499-1509

6. Luke, J. J., Bao, R., Sweis, R. F., Spranger, S. and Gajewski, T. F. (2019) WNT/ $\beta$-catenin pathway activation correlates with immune exclusion across human cancers. Clin. Cancer Res., 25, 3074-3083

7. Wang, B., Tian, T., Kalland, K.-H., Ke, X. and Qu, Y. (2018) Targeting $\mathrm{Wnt} / \beta$-catenin signaling for cancer immunotherapy. Trends Pharmacol. Sci., 39, 648-658

8. Haslam, A. and Prasad, V. (2019) Estimation of the percentage of US patients with cancer who are eligible for and respond to checkpoint inhibitor immunotherapy drugs. JAMA Netw. Open, 2, e192535

9. Khair, D. O., Bax, H. J., Mele, S., Crescioli, S., Pellizzari, G.,
Khiabany, A., Nakamura, M., Harris, R. J., French, E., Hoffmann, R. M., et al. (2019) Combining immune checkpoint inhibitors: established and emerging targets and strategies to improve outcomes in melanoma. Front. Immunol., 10, 453

10. Chen, D. S. and Mellman, I. (2013) Oncology meets immunology: the cancer-immunity cycle. Immunity, 39, 1-10

11. Binnewies, M., Roberts, E. W., Kersten, K., Chan, V., Fearon, D. F., Merad, M., Coussens, L. M., Gabrilovich, D. I., OstrandRosenberg, S., Hedrick, C. C., et al. (2018) Understanding the tumor immune microenvironment (TIME) for effective therapy. Nat. Med., 24, 541-550

12. Gonzalez, H., Hagerling, C. and Werb, Z. (2018) Roles of the immune system in cancer: from tumor initiation to metastatic progression. Genes Dev., 32, 1267-1284

13. Darvin, P., Toor, S. M., Sasidharan Nair, V. and Elkord, E. (2018) Immune checkpoint inhibitors: recent progress and potential biomarkers. Exp. Mol. Med., 50, 1-11

14. Szabo, C. I. and King, M. C. (1997) Population genetics of BRCAI and BRCA2. Am. J. Hum. Genet., 60, 1013-1020

15. Malone, K. E., Daling, J. R., Doody, D. R., Hsu, L., Bernstein, L., Coates, R. J., Marchbanks, P. A., Simon, M. S., McDonald, J. A., Norman, S. A., et al. (2006) Prevalence and predictors of BRCAI and BRCA2 mutations in a population-based study of breast cancer in white and black American women ages 35 to 64 years. Cancer Res., 66, 8297-8308

16. Bodmer, W. and Tomlinson, I. (2010) Rare genetic variants and the risk of cancer. Curr. Opin. Genet. Dev., 20, 262-267

17. Knudson, Jr., A. G. (1971) Mutation and cancer: statistical study of retinoblastoma. Proc. Natl. Acad. Sci. USA, 68, 820-823

18. Knudson, A. G. (2001) Two genetic hits (more or less) to cancer. Nat. Rev. Cancer, 1, 157-162

19. Donovan, S. L., Schweers, B., Martins, R., Johnson, D. and Dyer, M. A. (2006) Compensation by tumor suppressor genes during retinal development in mice and humans. BMC Biol., 4, 14

20. Ajioka, I., Martins, R. A. P., Bayazitov, I. T., Donovan, S., Johnson, D. A., Frase, S., Cicero, S. A., Boyd, K., Zakharenko, S. S. and Dyer, M. A. (2007) Differentiated horizontal interneurons clonally expand to form metastatic retinoblastoma in mice. Cell, 131, 378-390

21. Malkin, D., Li, F. P., Strong, L. C., Fraumeni, J. F. Jr, Nelson, C. E., Kim, D. H., Kassel, J., Gryka, M. A., Bischoff, F. Z., Tainsky, M. A., et al. (1990) Germ line p53 mutations in a familial syndrome of breast cancer, sarcomas, and other neoplasms. Science, 250, 1233 1238

22. Kemp, C. J., Wheldon, T. and Balmain, A. (1994) p53-deficient mice are extremely susceptible to radiation-induced tumorigenesis. Nat. Genet., 8, 66-69

23. Lee, J. M., Abrahamson, J. L. A., Kandel, R., Donehower, L. A. and Bernstein, A. (1994) Susceptibility to radiation-carcinogenesis and accumulation of chromosomal breakage in p53 deficient mice. Oncogene, 9, 3731-3736

24. Levine, A. J., Hu, W. and Feng, Z. (2006) The P53 pathway: what questions remain to be explored? Cell Death Differ., 13, 10271036 
25. Maistro, S., Teixeira, N., Encinas, G., Katayama, M. L. H., Niewiadonski, V. D. T., Cabral, L. G., Ribeiro, R. M., Gaburo Junior, N., de Gouvêa, A. C., Carraro, D. M., et al. (2016) Germline mutations in BRCA1 and BRCA2 in epithelial ovarian cancer patients in Brazil. BMC Cancer, 16, 934

26. Chan, S. H., Lim, W. K., Ishak, N. D. B., Li, S.-T., Goh, W. L., Tan, G. S., Lim, K. H., Teo, M., Young, C. N. C., Malik, S., et al. (2017) Germline mutations in cancer predisposition genes are frequent in sporadic sarcomas. Sci. Rep., 7, 10660

27. Kuchenbaecker, K. B., Hopper, J. L., Barnes, D. R., Phillips, K.A., Mooij, T. M., Roos-Blom, M.-J., Jervis, S., van Leeuwen, F. E., Milne, R. L., Andrieu, N., et al. (2017) Risks of breast, ovarian, and contralateral breast cancer for BRCA1 and BRCA2 mutation carriers. JAMA, 317, 2402-2416

28. Liaw, D., Marsh, D. J., Li, J., Dahia, P. L. M., Wang, S. I., Zheng, Z., Bose, S., Call, K. M., Tsou, H. C., Peacoke, M., et al. (1997) Germline mutations of the PTEN gene in Cowden disease, an inherited breast and thyroid cancer syndrome. Nat. Genet., 16, 6467

29. De Queiroz Rossanese, L. B., De Lima Marson, F. A., Ribeiro, J. D., Coy, C. S. and Bertuzzo, C. S. (2013) APC germline mutations in families with familial adenomatous polyposis. Oncol. Rep., 30, 2081-2088

30. Washington, K. and Zemper, A. E. D. (2019) Apc-related models of intestinal neoplasia: a brief review for pathologists. Surg. Exp. Pathol., 2, 11

31. Zeineldin, M. and Neufeld, K. L. (2013) More than two decades of Apc modeling in rodents. Biochim. Biophys. Acta, 1836, 80-89

32. Moore, L. E., Nickerson, M. L., Brennan, P., Toro, J. R., Jaeger, E., Rinsky, J., Han, S. S., Zaridze, D., Matveev, V., Janout, V., et al. (2011) Von hippel-lindau (VHL) inactivation in sporadic clear cell renal cancer: associations with germline $V H L$ polymorphisms and etiologic risk factors. PLoS Genet., 7, e1002312

33. Gray, P. N., Tsai, P., Chen, D., Wu, S., Hoo, J., Mu, W., Li, B., Vuong, H., Lu, H. M., Batth, N., et al. (2018) TumorNext-LynchMMR: a comprehensive next generation sequencing assay for the detection of germline and somatic mutations in genes associated with mismatch repair deficiency and Lynch syndrome. Oncotarget, 9, 20304-20322

34. Mou, H., Kennedy, Z., Anderson, D. G., Yin, H. and Xue, W. (2015) Precision cancer mouse models through genome editing with CRISPR-Cas9. Genome Med., 7, 53

35. Guernet, A. and Grumolato, L. (2017) CRISPR/Cas9 editing of the genome for cancer modeling. Methods, 121-122, 130-137

36. Park, S., Supek, F. and Lehner, B. (2018) Systematic discovery of germline cancer predisposition genes through the identification of somatic second hits. Nat. Commun., 9, 2601

37. Huang, K. L., Mashl, R. J., Wu, Y., Ritter, D. I., Wang, J., Oh, C., Paczkowska, M., Reynolds, S., Wyczalkowski, M. A., Oak, N., et al. (2018) Pathogenic germline variants in 10,389 adult cancers. Cell, 173, 355-370.e14

38. Kilpivaara, O. and Aaltonen, L. A. (2013) Diagnostic cancer genome sequencing and the contribution of germline variants. Science, 339, 1559-1562
39. Wang, E., Zaman, N., Mcgee, S., Milanese, J.-S., Masoudi-Nejad, A. and O'Connor-McCourt, M. (2015) Predictive genomics: a cancer hallmark network framework for predicting tumor clinical phenotypes using genome sequencing data. Semin. Cancer Biol., $30,4-12$

40. Sever, R. and Brugge, J. S. (2015) Signal transduction in cancer. Cold Spring Harb. Perspect. Med., 5, a006098-a006098

41. Wellenstein, M. D. and de Visser, K. E. (2018) Cancer-cellintrinsic mechanisms shaping the tumor immune landscape. Immunity, 48, 399-416

42. Nguyen, K. B. and Spranger, S. (2020) Modulation of the immune microenvironment by tumor-intrinsic oncogenic signaling. J. Cell Biol., 219, e201908224

43. Lim, Y. W., Chen-Harris, H., Mayba, O., Lianoglou, S., Wuster, A., Bhangale, T., Khan, Z., Mariathasan, S., Daemen, A., Reeder, J., et al. (2018) Germline genetic polymorphisms influence tumor gene expression and immune cell infiltration. Proc. Natl. Acad. Sci. USA, 115, E11701-E11710

44. Robinson, J., Halliwell, J. A., Hayhurst, J. D., Flicek, P., Parham, P. and Marsh, S. G. E. (2015) The IPD and IMGT/HLA database: allele variant databases. Nucleic Acids Res., 43, D423-D431

45. Marty, R., Kaabinejadian, S., Rossell, D., Slifker, M. J., van de Haar, J., Engin, H. B., de Prisco, N., Ideker, T., Hildebrand, W. H., Font-Burgada, J., et al. (2017) MHC-I genotype restricts the oncogenic mutational landscape. Cell, 171, 1272-1283.e15

46. Wang, E., Zou, J., Zaman, N., Beitel, L. K., Trifiro, M. and Paliouras, M. (2013) Cancer systems biology in the genome sequencing era: part 2, evolutionary dynamics of tumor clonal networks and drug resistance. Semin. Cancer Biol., 23, 286-292

47. Wang, E., Zou, J., Zaman, N., Beitel, L. K., Trifiro, M. and Paliouras, M. (2013) Cancer systems biology in the genome sequencing era: part 1, dissecting and modeling of tumor clones and their networks. Semin. Cancer Biol., 23, 279-285

48. Milanese J.-S., Tibiche C., Zou J., Meng Z., Nantel A., Drouin S., Marcotte R., and Wang E. (2019) Germline variants associated with leukocyte genes predict tumor recurrence in breast cancer patients. NPJ Precis. Oncol., 3, 28

49. Parsons, B. L. (2008) Many different tumor types have polyclonal tumor origin: evidence and implications. Mutat. Res., 659, 232247

50. Parsons, B. L. (2018) Multiclonal tumor origin: evidence and implications. Mutat. Res., 777, 1-18

51. Knudson, A. G. Jr. (1973) Mutation and human cancer. Adv. Cancer Res., 17, 317-352

52. Nowell, P. C. (1976) The clonal evolution of tumor cell populations. Science, 194, 23-28

53. Fearon, E. R., Hamilton, S. R. and Vogelstein, B. (1987) Clonal analysis of human colorectal tumors. Science, 238, 193-197

54. Vogelstein, B., Fearon, E. R., Hamilton, S. R. and Feinberg, A. P. (1985) Use of restriction fragment length polymorphisms to determine the clonal origin of human tumors. Science, 227, 642645

55. Ross, E. M. and Markowetz, F. (2016) OncoNEM: inferring tumor evolution from single-cell sequencing data. Genome Biol., 17, 69 
56. Hou, Y., Song, L., Zhu, P., Zhang, B., Tao, Y., Xu, X., Li, F., Wu, K., Liang, J., Shao, D., et al. (2012) Single-cell exome sequencing and monoclonal evolution of a JAK2-negative myeloproliferative neoplasm. Cell, 148, 873-885

57. Xu, X., Hou, Y., Yin, X., Bao, L., Tang, A., Song, L., Li, F., Tsang, S., Wu, K., Wu, H., et al. (2012) Single-cell exome sequencing reveals single-nucleotide mutation characteristics of a kidney tumor. Cell, 148, 886-895

58. Shi, X., Chakraborty, P. and Chaudhuri, A. (2018) Unmasking tumor heterogeneity and clonal evolution by single-cell analysis. J. Cancer Metastasis Treat., 4, 47

59. Milanese, J., Tibiche, C., Zaman, N., Zou, J., Han, P., Meng, Z., Nantel, A., Droit, A. and Wang, E. (2020) eTumorMetastasis, a network-based algorithm predicts clinical outcomes using wholeexome sequencing data of cancer patients. Genom. Proteom. Bioinfor., (In press)

60. Li, J., Lenferink, A. E. G., Deng, Y., Collins, C., Cui, Q., Purisima, E. O., O'Connor-McCourt, M. D. and Wang, E. (2010) Identification of high-quality cancer prognostic markers and metastasis network modules. Nat. Commun., 1, 34

61. Gao, S., Tibiche, C., Zou, J., Zaman, N., Trifiro, M., O'ConnorMcCourt, M. and Wang, E. (2016) Identification and construction of combinatory cancer hallmark-based gene signature sets to predict recurrence and chemotherapy benefit in stage II colorectal cancer. JAMA Oncol., 2, 37-45

62. Toi, M., Iwata, H., Yamanaka, T., Masuda, N., Ohno, S., Nakamura, S., Nakayama, T., Kashiwaba, M., Kamigaki, S. and Kuroi, K., et al. (2010) Clinical significance of the 21-gene signature (oncotype DX) in hormone receptor-positive early stage primary breast cancer in the Japanese population. Cancer, 116, 3112-3118

63. Feng, X., Xu, X., Li, D., Cui, Q. and Wang, E. (2019) Germline genomic patterns are associated with cancer risk, oncogenic pathways and clinical outcomes. bioRxiv, 616268

64. Xu, X., Li, J., Zou, J., Feng, X., Zhang, C., Zheng, R., Duanmu, W., Saha-Mandal, A., Ming, Z. and Wang, E. (2019) Association of germline variants in natural killer cells with tumor immune microenvironment subtypes, tumor-infiltrating lymphocytes, immunotherapy response, clinical outcomes, and cancer risk. JAMA Netw. Open, 2, e199292

65. Sharma, S., Kelly, T. K. and Jones, P. A. (2010) Epigenetics in cancer. Carcinogenesis, 31, 27-36

66. Witkowski, L., Carrot-Zhang, J., Albrecht, S., Fahiminiya, S., Hamel, N., Tomiak, E., Grynspan, D., Saloustros, E., Nadaf, J., Rivera, B., et al. (2014) Germline and somatic SMARCA4 mutations characterize small cell carcinoma of the ovary, hypercalcemic type. Nat. Genet., 46, 438-443

67. Butler, J. S., Koutelou, E., Schibler, A. C. and Dent, S. Y. R. (2012) Histone-modifying enzymes: regulators of developmental decisions and drivers of human disease. Epigenomics, 4, 163-177

68. Klutstein, M., Nejman, D., Greenfield, R. and Cedar, H. (2016) DNA methylation in cancer and aging. Cancer Res., 76, 34463450

69. Miao, D., Margolis, C. A., Gao, W., Voss, M. H., Li, W., Martini,
D. J., Norton, C., Bossé, D., Wankowicz, S. M., Cullen, D., et al. (2018) Genomic correlates of response to immune checkpoint therapies in clear cell renal cell carcinoma. Science, 359, 801-806

70. Pan, D., Kobayashi, A., Jiang, P., Ferrari de Andrade, L., Tay, R. E., Luoma, A. M., Tsoucas, D., Qiu, X., Lim, K., Rao, P., et al. (2018) A major chromatin regulator determines resistance of tumor cells to T cell-mediated killing. Science, 359, 770-775

71. Kearney, C. J., Vervoort, S. J., Hogg, S. J., Ramsbottom, K. M., Freeman, A. J., Lalaoui, N., Pijpers, L., Michie, J., Brown, K. K., Knight, D. A., et al. (2018) Tumor immune evasion arises through loss of TNF sensitivity. Sci. Immunol., 3, eaar3451

72. Beatty, G. L. and Paterson, Y. (2000) IFN- $\gamma$ can promote tumor evasion of the immune system in vivo by down-regulating cellular levels of an endogenous tumor antigen. J. Immunol., 165, 5502 5508

73. Houlahan, K. E., Shiah, Y.-J., Gusev, A., Yuan, J., Ahmed, M., Shetty, A., Ramanand, S. G., Yao, C. Q., Bell, C., O'Connor, E., et al. (2019) Genome-wide germline correlates of the epigenetic landscape of prostate cancer. Nat. Med., 25, 1615-1626

74. Foulkes, W. D. (2008) Inherited susceptibility to common cancers. N. Engl. J. Med., 359, 2143-2153

75. Torgovnick, A. and Schumacher, B. (2015) DNA repair mechanisms in cancer development and therapy. Front. Genet., 6, 157

76. Büttner, R., Longshore, J. W., López-Ríos, F., Merkelbach-Bruse, S., Normanno, N., Rouleau, E. and Penault-Llorca, F. (2019) Implementing TMB measurement in clinical practice: considerations on assay requirements. ESMO Open, 4, e000442

77. Caruso, C. (2019) TMB faces validation hurdles. Cancer Discov., 9, 1334-1334

78. Potapova, T. A., Zhu, J. and Li, R. (2013) Aneuploidy and chromosomal instability: a vicious cycle driving cellular evolution and cancer genome chaos. Cancer Metastasis Rev., 32, 377-389

79. Pérez de Castro, I. and Malumbres, M. (2012) Mitotic stress and chromosomal instability in cancer: the case for TPX2. Genes Cancer, 3, 721-730

80. Mackenzie, K. J., Carroll, P., Martin, C.-A., Murina, O., Fluteau, A., Simpson, D. J., Olova, N., Sutcliffe, H., Rainger, J. K., Leitch, A., et al. (2017) cGAS surveillance of micronuclei links genome instability to innate immunity. Nature, 548, 461-465

81. Motwani, M. and Fitzgerald, K. A. (2017) cGAS micro-manages genotoxic stress. Immunity, 47, 616-617

82. Breunis, W. B., Tarazona-Santos, E., Chen, R., Kiley, M., Rosenberg, S. A. and Chanock, S. J. (2008) Influence of cytotoxic T lymphocyte-associated antigen 4 (CTLA4) common polymorphisms on outcome in treatment of melanoma patients with CTLA-4 blockade. J. Immunother., 31, 586-590

83. Hamid, O., Schmidt, H., Nissan, A., Ridolfi, L., Aamdal, S., Hansson, J., Guida, M., Hyams, D. M., Gómez, H., Bastholt, L., et al. (2011) A prospective phase II trial exploring the association between tumor microenvironment biomarkers and clinical activity of ipilimumab in advanced melanoma. J. Transl. Med., 9, 204

84. Kuehn H. S., Ouyang W., Lo B., Deenick E. K., Niemela J. E., Avery D. T., Schickel J.-N., Tran D. Q., Stoddard J., Zhang Y., et al. (2014) Immune dysregulation in human subjects with 
heterozygous germline mutations in CTLA4. Science, 345,16231627

85. Chat, V., Ferguson, R., Simpson, D., Kazlow, E., Lax, R., Moran, U., Wilson, M., Pavlick, A. C., Sullivan, R. J., Flaherty, K., et al. (2018) Autoimmune genetic variants as germline biomarkers of response in melanoma immunotherapy treatment. J. Clin. Oncol., 36, 3079-3079

86. Ugurel, S., Schrama, D., Keller, G., Schadendorf, D., Bröcker, E.B., Houben, R., Zapatka, M., Fink, W., Kaufman, H. L. and Becker, J. C. (2008) Impact of the CCR5 gene polymorphism on the survival of metastatic melanoma patients receiving immunotherapy. Cancer Immunol. Immunother., 57, 685-691

87. Uccellini, L., De Giorgi, V., Zhao, Y., Tumaini, B., Erdenebileg, N., Dudley, M. E., Tomei, S., Bedognetti, D., Ascierto, M. L., Liu, Q., et al. (2012) IRF5 gene polymorphisms in melanoma. J. Transl. Med., 10, 170

88. Arce Vargas, F., Furness, A. J. S., Litchfield, K., Joshi, K., Rosenthal, R., Ghorani, E., Solomon, I., Lesko, M. H., Ruef, N., Roddie, C., et al. (2018) Fc effector function contributes to the activity of human anti-CTLA-4 antibodies. Cancer Cell, 33, 649663.e4

89. Van Allen, E. M., Golay, H. G., Liu, Y., Koyama, S., Wong, K., Taylor-Weiner, A., Giannakis, M., Harden, M., Rojas-Rudilla, V., Chevalier, A., et al. (2015) Long-term benefit of PD-L1 blockade in lung cancer associated with JAK3 activation. Cancer Immunol. Res., 3, 855-863

90. Zitvogel, L., Kepp, O. and Kroemer, G. (2011) Immune parameters affecting the efficacy of chemotherapeutic regimens. Nat. Rev. Clin. Oncol., 8, 151-160

91. Vacchelli E., Ma Y., Baracco E. E., Sistigu A., Enot D. P., Pietrocola F., Yang H., Adjemian S., Chaba K., Semeraro M., et al.
(2015) Chemotherapy-induced antitumor immunity requires formyl peptide receptor 1 . Science, 350, 972-978

92. Lamichhane, P., Karyampudi, L., Shreeder, B., Krempski, J., Bahr, D., Daum, J., Kalli, K. R., Goode, E. L., Block, M. S., Cannon, M. J., et al. (2017) IL10 Release upon PD-1 blockade sustains immunosuppression in ovarian cancer. Cancer Res., 77, 66676678

93. Zaretsky, J. M., Garcia-Diaz, A., Shin, D. S., Escuin-Ordinas, H., Hugo, W., Hu-Lieskovan, S., Torrejon, D. Y., Abril-Rodriguez, G., Sandoval, S., Barthly, L., et al. (2016) Mutations associated with acquired resistance to PD-1 blockade in melanoma. N. Engl. J. Med., 375, 819-829

94. Gao, J., Shi, L. Z., Zhao, H., Chen, J., Xiong, L., He, Q., Chen, T., Roszik, J., Bernatchez, C., Woodman, S. E., et al. (2016) Loss of IFN- $\gamma$ pathway genes in tumor cells as a mechanism of resistance to anti-CTLA-4 therapy. Cell, 167, 397-404.e9

95. Shan, J., Chouchane, A., Mokrab, Y., Saad, M., Boujassoum, S., Sayaman, R. W., Ziv, E., Bouaouina, N., Remadi, Y., Gabbouj, S., et al. (2019) Genetic variation in CCL5 signaling genes and triple negative breast cancer: susceptibility and prognosis implications. Front. Oncol., 9, 1328

96. Hosseini, E., Schwarer, A. P. and Ghasemzadeh, M. (2015) Do human leukocyte antigen E polymorphisms influence graft-versusleukemia after allogeneic hematopoietic stem cell transplantation? Exp. Hematol., 43, 149-157

97. Wu, S., Powers, S., Zhu, W. and Hannun, Y. A. (2016) Substantial contribution of extrinsic risk factors to cancer development. Nature, 529, 43-47

98. Tomasetti, C., Li, L. and Vogelstein, B. (2017) Stem cell divisions, somatic mutations, cancer etiology, and cancer prevention. Science, $355,1330-1334$ 